Archives de sciences sociales des religions

$162 \mid 2013$

L'orthodoxie russe aujourd'hui | Varia

\title{
Introduction : l'orthodoxie russe aujourd'hui
}

\section{Kathy Rousselet}

\section{OpenEdition}

Journals

Édition électronique

URL : http://journals.openedition.org/assr/25023

DOI : 10.4000/assr.25023

ISSN : 1777-5825

Éditeur

Éditions de l'EHESS

Édition imprimée

Date de publication : 1 juillet 2013

Pagination : 9-14

ISBN : 978-2-71322395-2

ISSN : 0335-5985

Référence électronique

Kathy Rousselet, «Introduction : I'orthodoxie russe aujourd'hui », Archives de sciences sociales des religions [En ligne], 162 | 2013, mis en ligne le 01 juillet 2016, consulté le 22 mars 2021. URL : http:// journals.openedition.org/assr/25023; DOI : https://doi.org/10.4000/assr.25023 


\section{Kathy Rousselet}

\section{Introduction : l'orthodoxie russe aujourd'hui}

Tous les observateurs le constatent : l'Église orthodoxe russe est devenue de plus en plus visible dans la sphère publique et certains analystes parlent d'une " désécularisation » à l'œuvre dans le pays (V. Karpov, 2010). Pourtant, les derniers sondages attestent d'une baisse d'intérêt pour le religieux. Selon le centre Levada, si en $200980 \%$ des personnes interrogées se disaient appartenir à l'Église orthodoxe russe, en novembre 2012, ils ne sont plus que $74 \%{ }^{1}$. Un sondage effectué en 2012 par l'institut Sreda sur un échantillon de 56900 personnes dans l'ensemble des régions de Russie conclut que seulement $41 \%$ des personnes interrogées se déclarent orthodoxes dans l'Église ${ }^{2}$. Selon cette même étude, $16 \%$ de l'ensemble des personnes interrogées et $29 \%$ des personnes se disant orthodoxes font confiance au patriarche. Ces résultats sont certes en partie la conséquence immédiate de la vague de protestations sociopolitiques qu'a connue le pays en 2011-2012. Cette vague a touché la hiérarchie de l'Église, tant celle-ci est liée au pouvoir politique; plusieurs scandales l'ont éclaboussée et l'affaire du groupe Pussy Riot a provoqué un divorce entre l'Église et une partie de l'intelligentsia. Mais la désaffection pour l'Église n'est pas que conjoncturelle. Des observateurs au sein de l'Église affirment aujourd'hui que la période qui a reçu le nom de renaissance religieuse est terminée (S. Čapnin, 2013). Début 2011, constatant que les deux-tiers des enfants de personnes engagées dans l'Église l'ont quittée, un membre de l'Église, l'higoumène Piotr Méchtchérinov expliquait cette évolution entre autres par le fait que la religiosité des familles postsoviétiques ne correspondrait souvent en rien à la tradition même de l'orthodoxie ; loin d'avoir un contenu chrétien, elle serait un "mélange sui generis d'idéologie, de magie et de complexes “soviétiques”, mimant les mœurs orthodoxes (l'absence de sens des responsabilités sous couvert d'“obéissance religieuse", l'absence de respect à l'égard de soi et d'autrui sous couvert d' "humilité religieuse", le conflit et la méchanceté sous couvert de "lutte pour la pureté

1. Voir http://www.levada.ru/17-12-2012/v-rossii-74-pravoslavnykh-i-7-musulman, consulté le 16 mars 2013.

2. Les résultats de ce sondage ont été publiés dans Atlas religij i nacional'nostej Rossijskoj Federacii (2012). 
de l'orthodoxie", etc.) ". L'absence d'attrait de l'Église auprès des jeunes serait largement due aux faiblesses de son message actuel (P. Meŝerinov, 2011).

De fait, le contexte post-soviétique a joué une influence complexe sur le développement du religieux. Si, à la fin des années 1980, la religion était avant tout synonyme de libération politique et d'éthique, peu à peu sa forme institutionnalisée a avant tout contribué à alimenter un système politique unanimiste (K. Rousselet, 2013). Les élites religieuses sont dominées par des intérêts politiques et économiques. Quant au sentiment religieux, il s'est développé de façon très contrastée. Le sentiment d'incertitude lié aux rapides changements socioéconomiques, la démoralisation et la quête de repères existentiels sont sans nul doute à l'origine des nombreuses conversions à l'âge adulte ; elles ont aussi provoqué l'apparition de guides spirituels de toutes sortes. En l'absence d'une transmission contrôlée de la foi, des messages catastrophistes se sont propagés. L'orthodoxie prend les formes les plus diverses. La religion orthodoxe dans sa tendance la plus conservatrice est portée par les monastères, mais aussi les laisséspour-compte de la société russe, nostalgiques d'un ordre moral soviétique et hostiles aux valeurs dites occidentales. Des courants, minoritaires, appréciés par une partie de l'intelligentsia, dialoguent avec la modernité.

Tout comme la société russe de façon générale, le paysage religieux est particulièrement divers. La différenciation entre villes et campagnes se combine à de grandes variations régionales. Les zones rurales sont à la fois dépeuplées et plus déchristianisées que les zones urbaines. D'après le sondage de l'institut Sreda, les régions de Sibérie sont plus fortement marquées par l'athéisme que le reste de la Russie : dans le Primorié, le pourcentage de personnes qui se disent athées atteint les $35 \%$ (contre $13 \%$ en moyenne nationale). L'orthodoxie se développe dans un climat individualiste dans les grandes villes qui connaissent un développement proche de celui observé en Europe occidentale, tandis qu'elle nourrit des tendances identitaires, par exemple, dans les milieux cosaques, dans le sud de la Russie. C'est dans cette région très conservatrice et que l'on a appelée la ceinture rouge, car le communisme y fut le plus tenace dans les années 1990, que le pourcentage de ceux qui se disent appartenir à l'Église orthodoxe est un des plus forts. De façon générale, la pratique religieuse est très faible. D’après le même sondage effectué en 2012 par l'institut Sreda, en moyenne, $2 \%$ des personnes interrogées disent se confesser au moins une fois par mois, $3 \%$ disent participer à la vie de la communauté paroissiale.

On conclurait volontiers, au vu des tendances observées, que la situation religieuse russe converge, au moins en partie, avec celle qui prévaut dans les sociétés occidentales. On y observe la même dérégulation institutionnelle, le même essor d'une religion hors des murs de l'Église. De nombreux néophytes préfèrent à la fréquentation des paroisses les pèlerinages sur les lieux saints (entre autres, J. Kormina, 2010, 2013) ou la visite des foires orthodoxes (I. Naletova, 
$2005)$; les forums Internet en attirent d'autres. Pourtant, cette faiblesse institutionnelle semble aussi propre au fonctionnement de la religion orthodoxe, tel qu'on l'observe déjà à la période tsariste (V. Shevzov, 2004). Elle s'inscrit aussi dans le prolongement des conditions imposées par le système soviétique, dans le sens précisément d'une non-institutionnalisation; la religion est alors aussi transmise par des prêtres et des membres de l'Église des catacombes, par des intellectuels, mais également et surtout par des femmes âgées présentes dans les quelques églises en activité ou en dehors d'elles (A. Agadjanian, K. Rousselet, 2010). La religion se conserve alors largement par la mémoire des gestes et l'adhésion religieuse se définit par la capacité de les reproduire le plus fidèlement possible. Les tendances actuelles, et en particulier la privatisation du religieux ne sont pas que le fruit de la sécularisation; elles sont aussi le produit d'une histoire longue, bien particulière.

Dans le prolongement d'études antérieures (A. Agadjanian et K. Rousselet 2005, 2006, 2011 ; Ž. Kormina, A. Pančenko, S. Štyrkov, 2006 ; C. Hann and H. Goltz, 2010), c'est à une analyse des pratiques religieuses, aussi bien au sein des structures ecclésiales qu'en dehors d'elles, qu'est consacré ce dossier des Archives de sciences sociales des religions. Il part de la conviction que ce n'est qu'à travers une observation et une description précise de ce qui se fait, de la religion vécue, que l'on peut comprendre les évolutions de la vie orthodoxe aujourd'hui. C'est au niveau le plus microsociologique que se saisissent les fils de continuité d'une histoire marquée pourtant par de forts bouleversements institutionnels. Plusieurs questions s'y entrecroisent.

La première porte sur l'autorité et la concurrence religieuses, ce à différents niveaux. Les détenteurs non institutionnels de l'époque soviétique entrent en conflit avec le personnel clérical recruté en nombre après 1988 pour fixer les critères d'authenticité des dogmes et des pratiques. Dans un souci de contrôle des ressources économiques et de régulation du croire, la hiérarchie ecclésiastique œuvre à la centralisation du pouvoir religieux, la réforme des statuts paroissiaux en étant une des dernières étapes. L'éducation religieuse et la conception de manuels catéchétiques deviennent un enjeu majeur. Mais la discipline bureaucratique n'empêche pas un fort localisme et la concentration religieuse autour de personnes charismatiques qui échappent largement à l'institution. S'agissant des startsy, virtuoses religieux et directeurs spirituels, Jeanne Kormina rappelle qu'il est souvent difficile de distinguer les vrais des faux. Les pratiques et les discours les plus divers entourent leur définition. L'anthropologue analyse, dans ce dossier, la fabrication du charisme, sur l'île Zalita, près de la frontière avec la Lettonie, de Nikolaï Gourianov (1909-2002), respecté comme symbole de l'authenticité de la Russie et porteur de l'identité nationale. Elle distingue plusieurs catégories d'entrepreneurs religieux, dont des orthodoxes fondamentalistes, luttant pour sa canonisation comme pour celle de Grigori Raspoutine, mystique et guérisseur devenu proche conseiller de la famille impériale. 
Les prêtres exercent une autorité personnalisée sur les laïcs. Celle-ci, loin d'alimenter une régulation du croire en termes d'homogénéisation, contribue à sa diversité. Elle peut se manifester en dehors de la vie paroissiale stricto sensu. Pourtant, paradoxalement, cette diversité n'est pas considérée comme une faiblesse interne de l'Église, bien au contraire. Dans son étude anthropologique sur les hommes d'affaires dans la région de Vladimir, Tobias Köllner souligne en particulier que les prêtres privilégient la rectitude non des croyances, mais des actions rituelles, ceci assurant de la "flexibilité » à l'orthodoxie russe et une adaptation de celle-ci aux besoins de chacun. Il ajoute qu' "ainsi, la religion pratiquée par les hommes d'affaires n'entre pas en contradiction avec les doctrines orthodoxes et permet d'intégrer ces entrepreneurs dans la communauté orthodoxe. " La relation au prêtre permet de surmonter la disjonction entre sentiment d'appartenance et liberté de croyance.

Face à une logique, très marquée, de privatisation et d'individualisation du religieux, en affinité avec des paroisses d'usagers, ou plus exactement de croyants de passage (zahožane), se constituent, ici ou là, de solides communautés, formées d'orthodoxes engagés, ayant choisi leur paroisse non par proximité spatiale mais en fonction du prêtre du lieu, qui assure aussi leur direction spirituelle. Elles développent souvent une forte activité dans le monde (aide aux prisonniers, assistance dans les hôpitaux...) et contribuent à la reconstitution du tissu social, mis à mal par les bouleversements des années 1990. L'Union des fraternités de la Transfiguration du père Kotchetkov ou la paroisse Saints-Côme-et-Damien constituée par des disciples du père Alexandre Men en sont deux archétypes, étudiés ici par Alexandre Agadjanian. L'une et l'autre insistent sur le sentiment communautaire et valorisent les petits groupes de type familial, même si leurs modes d'organisation sont assez différents ; l'Union des fraternités de la Transfiguration privilégie l'homogénéité du groupe, la paroisse Saints-Côme-et-Damien se montrant plus ouverte à la diversité. Elles subissent l'empreinte de leurs pasteurs dont les parcours religieux sont très différents.

Une autre question porte sur les acteurs mêmes de cette vie religieuse. L'étude sur le fonctionnement des paroisses montre comment l'engagement dans le système soviétique, et en particulier dans le Komsomol, organisation de la jeunesse communiste, est souvent un préalable à un engagement actif dans l'Église (Kathy Rousselet). Plus que jamais, le séculier et le religieux s'entremêlent, et le récit de vie privilégie la continuité. Étudiant les femmes dans l'éducation religieuse dans deux villes de régions différentes, Detelina Tocheva et Agata Ładykowska soulignent également que celles-ci, formées à la pédagogie dans le système soviétique, éventuellement même à l'époque professeurs d'athéisme, sont employées par les prêtres parce que ce sont de bonnes «techniciennes de l'éducation » et qu'elles ont le sens de l'organisation.

Ce dossier bouleverse peut-être certaines idées reçues. Il souligne le caractère singulier du développement du religieux en Russie, dû au contexte post-soviétique, 
mais aussi aux spécificités de la religion orthodoxe. La vie religieuse échappe largement aux relations entre le Patriarcat et l'État. Les tendances autoritaires à l'intérieur de l'Église n'empêchent pas une pluralité de pratiques au niveau local. Les engagements religieux d'aujourd'hui s'inscrivent dans le prolongement des engagements sécularisés d'hier. On a volontiers centré les analyses sur le fait religieux en Russie autour d'une rupture avec la situation précédente. Les observateurs ont voulu y trouver des traits communs à d'autres contextes, que ce soit en termes de sécularisation ou désécularisation, en lien avec une modernité post-soviétique qui reste néanmoins difficile à définir. Si l'approche comparative est sans nul doute pertinente, l'heure semble venue de regarder également les choses de plus près afin de mieux spécifier.

Kathy ROUSSELET

Sciences Po, Centre d'études et de recherches internationales

kathy.rousselet@mac.com

\section{Bibliographie}

Agadjanian Alexandre, Rousselet Kathy (dir.), 2005, "Les pratiques religieuses en Russie ", Revue d'études comparatives Est-Ouest.

- (dir.), 2006, Religioznye praktiki v sovremennoj Rossii [Les pratiques religieuses dans la Russie contemporaine], Moscou, Novoe Izdatel'stvo, 2006.

-, 2010, "Individual and Collective Identities in Russian Orthodoxy ", in Hann C. and Goltz H. (éds.), Orthodoxy, Orthopraxy, Parádosis: Eastern Christians in Anthropological Perspective, Berkeley, California University Press, p. 311-328.

- (dir.), 2011, Prihod i obŝina [La paroisse et la communauté], Moskva, Izd. Ves’ Mir.

AtLAS RELIGIJ I NACIONAL'NOSTEJ RossijSKOJ FEDERACII [Atlas des religions et des nationalités de la Fédération de Russie], 2012, http://sreda.org/arena, consulté le 16 mars 2013.

ČapnIN Sergej, 2013, Cerkov'v postsovetskoj Rossii. Vozroždenie, kačestvo very, dialog s obŝestvom. Sub"ektivnye razmyšleniâ o nastoâŝem i buduŝem [L'Église dans la Russie post-soviétique. Renaissance, qualité de la foi, dialogue avec la société. Réflexions subjectives sur le présent et l'avenir], Moscou, Arefa.

Hann Christopher, Goltz Hermann (éds.), 2010, Orthodoxy, Orthopraxy, Parádosis: Eastern Christians in Anthropological Perspective, Berkeley, California University Press.

KARPov Vyacheslav, 2010, "Desecularization: A Conceptual Framework », Journal of Church and State, 52 (2), p. 232-270.

Kormina Žanna, PANČENKo Aleksandr, ŠTYRKov Sergej, 2006, Sny Bogorodicy. Issledovaniâ po antropologii religii [Les rêves de la Vierge. Études d'anthropologie religieuse], Sankt-Peterburg, Izd. Evropejskogo Universiteta.

Kormina Jeanne, 2010, « Avtobusniki: Russian Orthodox Pilgrims’ Longing for Authenticity ", in Hann C. and Goltz H. (éds.), Orthodoxy, Orthopraxy, Parádosis: Eastern Christians in Anthropological Perspective, Berkeley, California University Press, p. 267286. 
Kormina Jeanne, 2013, "La langue des pèlerins orthodoxes : l'“énergie sacrale d'un lieu chargé de prière" ", Slavica Occitania, n 36 ( "Pèlerinages en Eurasie et au-delà ») (à paraître).

MeŜERINOv Petr, "Počemu deti vocerkovlennyh roditelej uhodât iz Cerkvi » [Pourquoi les enfants de personnes engagées dans l'Église la quittent], 9 février 2011 (consulté le 16 avril 2013), http://www.pravmir.ru/pochemu-deti-vocerkovlennyx-roditelejuxodyat-iz-cerkvi/

NALETOVA Inna, 2006, «Sovremennye pravoslavnye ârmarki kak vyraženie pravoslavnoj very vne hrama " [Les foires orthodoxes aujourd'hui comme expression de la foi orthodoxe en dehors de l'église], in A. Agadjanian, K. Rousselet (dir.), Religioznye praktiki v sovremennoj Rossii [Les pratiques religieuses dans la Russie contemporaine], Moscou, Novoe Izdatel'stvo, p. 178-198.

Rousselet Kathy, 2013, "Sécularisation et orthodoxie dans la Russie contemporaine : pour une hypothèse continuiste ? ", Questions de recherche, no 42, mai, Paris, Sciences Po, Centre d'études et de recherches internationales.

Shevzov Vera, 2004, Russian Orthodoxy of the Eve of Revolution, Oxford University Press. 